\title{
Synthesis of Novel Pyridopyridazin-3(2H)-one Derivatives and Evaluation of Their Cytotoxic Activity against MCF-7 Cells
}

\author{
Periasamy Selvakumar, ${ }^{1}$ Sathiah Thennarasu, ${ }^{1}$ and Asit Baran Mandal ${ }^{2}$ \\ ${ }^{1}$ Organic Chemistry Division, CSIR-Central Leather Research Institute, Adyar, Chennai 600 020, India \\ ${ }^{2}$ Chemical Laboratory, CSIR-Central Leather Research Institute, Adyar, Chennai 600 020, India \\ Correspondence should be addressed to Sathiah Thennarasu; thennarasu@gmail.com \\ and Asit Baran Mandal; abmandal@hotmail.com
}

Received 28 October 2013; Accepted 13 February 2014; Published 22 April 2014

Academic Editors: T. H. Al-Tel, F. Dufrasne, and C. Gong

Copyright (C) 2014 Periasamy Selvakumar et al. This is an open access article distributed under the Creative Commons Attribution License, which permits unrestricted use, distribution, and reproduction in any medium, provided the original work is properly cited.

\begin{abstract}
A series of pyridopyridazin-3(2H)-one derivatives was synthesized in two facile steps. Mannich-type three-component condensation afforded the 2,6-diaryl piperidin-4-one derivatives, which underwent intramolecular cyclization in the presence of hydrazine or phenylhydrazine to yield the corresponding pyridopyridazin-3(2H)-one derivatives. All the derivatives of pyridopyridazin$3(2 \mathrm{H})$-one, except $\mathbf{3 e}$ and $\mathbf{3 f}$, showed moderate activity against human breast adenocarcinoma (MCF-7) cells. The higher degree of inhibition of MCF-7 cell proliferation shown by $\mathbf{2 a - 2} \mathbf{f}$ indicates the significance of the amide proton in pyridopyridazin-3(2H)-one derivatives.
\end{abstract}

\section{Introduction}

Among the nitrogen containing six-member heterocylic compounds, the piperidine structural motif is often found in naturally occurring bioactive compounds such as alkaloids [1]. Piperidin-3-one derivatives are used as precursors for the synthesis of antimalarial agents, febrifugine, and isofebrifugine [2]. Piperidin-4-ones mostly display varied and potent biological properties such as antiviral, antitumour, analgesic, local anaesthetic, antimicrobial, fungicidal, herbicidal, insecticidal, antihistaminic, anti-inflammatory, anticancer, CNS stimulant, and depressant properties[3-9]. Recent reports suggest that compounds containing the piperidin-4-one moiety elicit excellent biological activities when aromatic substitutions are present at 2 and/or 6 positions [10].

Another pharmacologically important heterocyclic structural motif is the pyridazin- $3(2 \mathrm{H})$-one unit, which has been found to inhibit the activities of cGMP-phosphodiesterase (PDE3) and cAMP-phosphodiesterase (PDE4) enzymes [11]. Also, pyridazin- $3(2 \mathrm{H})$-one possesses different pharmacological activities like analgesic, anti-inflammatory, antibacterial, herbicidal, antifungal, antituberculotic, anti-AIDs, antitumour, antihypertensive, anticonvulsant, and antiviral activities [12-16]. Besides these activities, the polyfunctional tetrahydro-2H-pyrano[3,2-c]pyridazin-3(6H)-one derivatives have been shown to act as potent anticancer agents [17]. Recently, a series of pyridazinone derivatives bearing benzenesulfonamide moiety have also been reported to act as anticancer agents [18].

Mannich-type condensation involving a ketone having two active methylene groups, an aromatic aldehyde, and ammonium acetate, resulting in the formation of 2,6diarylpiperidin-4-one, was first reported by Noller and Baliah [19]. Formation of 2,6-diarylpiperidin-4-one derivatives from the condensation of aryl aldehyde, ammonia, and levulinic acid or its ethyl ester was also observed by Baliah and Ekambaram [20]. However, a mixture of 2,6-diarylpiperidin-4-one and 1,9-diazabicyclononane derivatives was obtained from the condensation of acetone, aryl aldehyde, and ammonia [21]. Later on, Baliah and Jeyaraman reported a similar condensation involving cyclic ketones resulting in the formation of different azabicyclononanes [22]. Recently, Thennarasu and Perumal have reported the formation of 3-pentyl-2,6diphenylpiperidine-4-one in moderate yield via Mannichtype condensation of octan-2-one, benzaldehyde, and ammonium acetate in absolute ethanol [23]. 
We, herein, report an improved method for the synthesis and complete characterization of different 4-oxo-2,6-diphenylpiperidin-3-yl-acetates (Scheme 1). Subsequent cyclization of these derivatives into the corresponding pyrido[4,3c]pyridazin-3(2H)-one derivatives using hydrazine and phenylhydrazine (Scheme 2) is also reported for the first time. In addition, the anticancer activity of these new pyrido[4,3-c]pyridazin-3(2H)-one derivatives is presented.

\section{Results and Discussion}

2.1. Chemistry. Three-component condensation of benzaldehyde, ammonium acetate, and ethyl levulinate in methanol at $\sim 60^{\circ} \mathrm{C}$ afforded ethyl 4-oxo 2,6-diphenylpiperidin-3yl acetate in moderate yield (56\%). After optimizing the reaction conditions (temperature, time, and amount of catalyst $\mathrm{AcOH}$ ), various aryl aldehydes were allowed to react with ethyl levulinate and ammonium acetate in methanol-acetic acid medium at $\sim 60^{\circ} \mathrm{C}$ to afford the corresponding ethyl 4-oxo2,6-diarylpiperidin-3yl acetate derivatives in good yields. Typically, when benzaldehyde was used and the reaction was carried out in $70 \mathrm{~mol} \%$ glacial acetic acid in methanol, the yield improved from $56 \%$ to $77 \%$.

All the synthesized compounds were characterized using IR and NMR and ESI-MS techniques. For instance, the IR spectrum of compound la displays the secondary amine $\mathrm{NH}$ stretching at $3425 \mathrm{~cm}^{-1}$. The ketone and ester carbonyl vibrations occur, respectively, at $1723 \mathrm{~cm}^{-1}$ and $1654 \mathrm{~cm}^{-1}$ and indicate the formation of a cyclic ketone. The ${ }^{1} \mathrm{H}$ NMR spectrum of 1a shows a broad peak at $\sim 2.09$ ppm that can be ascribed to the $\mathrm{NH}$ proton of a secondary amine. $\mathrm{D}_{2} \mathrm{O}$ induced proton exchange diminishes the intensity of the peak at $\sim 2.09$ ppm confirming the exchangeability of the NH proton. All other protons were assigned with the aid of published literature [20]. The ketone and ester carbonyl resonances at 207.8 and $\sim 172.3$ ppm, respectively, in the ${ }^{13} \mathrm{C}$ NMR spectrum of la confirm the presence of the heterocyclic compound 1a. The formation of la was further confirmed using ESIMS data. Similarly, the structures of 2,6-diarylpiperidin-4one derivatives $\mathbf{1 b}-\mathbf{f}$ were also confirmed using mass and spectroscopic data (see Supplementary Material available online at http://dx.doi.org/10.1155/2014/410716).

In view of the wide spectrum of biological activities documented for piperidine and pyridazinone pharmacophores, we reasoned that the combination of these two different pharmacophores into a single structural scaffold would confer synergistic properties on the new molecule. Accordingly, we synthesized pyrido[4,3-c]pyridazin-3(2H)one derivatives using hydrazine and phenylhydrazine mediated cyclization as depicted in Scheme 2.

The reaction of hydrazine hydrate with ethyl 4-oxo 2,6diarylpiperidin-3yl acetates in ethanol under reflux condition yielded in due course the corresponding 4,4a,5,6,7,8hexahydro-5,7-diarylpyrido[4,3-c]pyridazin-3(2H)-one derivatives $\mathbf{2 a - f}$. Under identical reaction conditions, phenylhydrazine failed to yield the corresponding $4,4 \mathrm{a}, 5,6,7,8$ hexahydro-2,5,7-triarylpyrido[4,3-c]pyridazin-3(2H)-one derivatives $3 \mathbf{a}-\mathbf{f}$. However, the reaction was accomplished in dry toluene containing $20 \mathrm{~mol} \%$ trifluoroacetic acid with moderate to good yields (Scheme 2) [24].

The structures of 4,4a,5,6,7,8-hexahydro-5,7-diarylpyrido[4,3-c]pyridazin-3(2H)-one derivatives $2 \mathbf{a}-\mathbf{f}$ were confirmed using spectroscopic data. The two bands at $3371 \mathrm{~cm}^{-1}$ and $3281 \mathrm{~cm}^{-1}$ in the IR spectrum of the compound $\mathbf{2 a}$ could be assigned to the secondary amine $\mathrm{NH}$ and amide $\mathrm{NH}$ stretching vibrations, respectively. This observation is corroborated by the diminished intensity of amine $\mathrm{NH}$ peak at $\sim 2.1 \mathrm{ppm}$ and the amide $\mathrm{NH}$ peak at $\sim 8.7 \mathrm{ppm}$ in the ${ }^{1} \mathrm{H}$ NMR spectrum of $\mathbf{2 a}$ obtained after proton exchange with $\mathrm{D}_{2} \mathrm{O}$. The strong amide carbonyl stretching at $1685 \mathrm{~cm}^{-1}$ substantiates the formation of pyrido[4,3-c]pyridazin-3(2H)one structure. The appearance of amide carbon peak at $166 \mathrm{ppm}$ and imine carbon peak at $\sim 152 \mathrm{ppm}$ and the absence of parent-ketone peak at $\sim 207 \mathrm{ppm}$ in the ${ }^{13} \mathrm{C}$ NMR spectrum provide complementary evidence to confirm the formation of compound $\mathbf{2 a}$.

The structural elucidation of $4,4 \mathrm{a}, 5,6,7,8$-hexahydro2,5,7-triarylpyrido[4,3-c]pyridazin-3(2H)-one derivatives 3a-f was achieved through spectral analysis. The appearance of amine $\mathrm{NH}$ band at $~ 3320 \mathrm{~cm}^{-1}$ and absence of amide $\mathrm{NH}$ band at $\sim 3281 \mathrm{~cm}^{-1}$ in the IR spectrum of the compound $\mathbf{3 b}$ are an indication of phenyl substitution at amide nitrogen of 3b. This proposition is supported by the observation of only the amine $\mathrm{NH}$ peak at $\sim 2.08 \mathrm{ppm}$ and the absence of any amide $\mathrm{NH}$ resonances in the ${ }^{1} \mathrm{H}$ NMR spectrum of $\mathbf{3 b}$. The strong amide carbonyl stretching at $\sim 1688 \mathrm{~cm}^{-1}$ is evidence to the formation of $\mathbf{3 b}$. The amide carbon resonance at $\sim 164 \mathrm{ppm}$ and imine carbon resonance at $\sim 153 \mathrm{ppm}$ in the ${ }^{13} \mathrm{C}$ NMR spectrum confirms the formation of compound $3 b$.

Several conformational isomers are possible for $\mathbf{1 a}-\mathbf{f}, \mathbf{2} \mathbf{a}-$ $\mathbf{f}$, and 3a-f depending on the nature of the substituents and the reaction conditions used for their syntheses. Therefore, it was important to determine the preferred types of chair or boat conformations of $\mathbf{1 a}-\mathbf{f}, \mathbf{2} \mathbf{a}-\mathbf{f}$, and $\mathbf{3 a - f}$. The conformational assignments were investigated using ${ }^{13} \mathrm{C}$ NMR (chemical shifts) and ${ }^{1} \mathrm{H}$ NMR (vicinal coupling constants) spectra [25-28]. The dihedral angles derived from the vicinal coupling constant values obtained from the ${ }^{1} \mathrm{H}$ NMR spectrum of $\mathbf{3 b}$ suggested a chair conformation to the piperidine ring and a distorted/half-chair conformation to the pyridazine ring. This conformation of $\mathbf{3} \mathbf{b}$ was further confirmed through single crystal XRD analysis (Figure 1) [29].

2.2. Anticancer Activity. The newly synthesized pyridopyridazin-3(2H)-one derivatives $\mathbf{2} \mathbf{a}-\mathbf{f}$ and $\mathbf{3 a - f}$ were evaluated for anticancer activity in vitro against MCF-7 breast cancer cells. Doxorubicin was used as the standard drug. MTT assay showed that all the pyridopyridazin-3(2H)-one derivatives 2a-f and 3a-f possessed moderate cytotoxic activity (Table 1) $[30,31]$. Only the compounds $3 \mathbf{e}(\sim 810 \mu \mathrm{M})$ and $3 \mathbf{f}(\sim$ $473 \mu \mathrm{M})$ exhibited weak activity against the cancer cell line. A comparison of the anticancer activities of $\mathbf{2 a - f}$ and $\mathbf{3 a}-\mathbf{f}$ reveals that only the three compounds $\mathbf{2 d - f}$ bearing electron withdrawing substituents in the aromatic ring show the highest activity. It is also imperative to note that only the hydrazine 


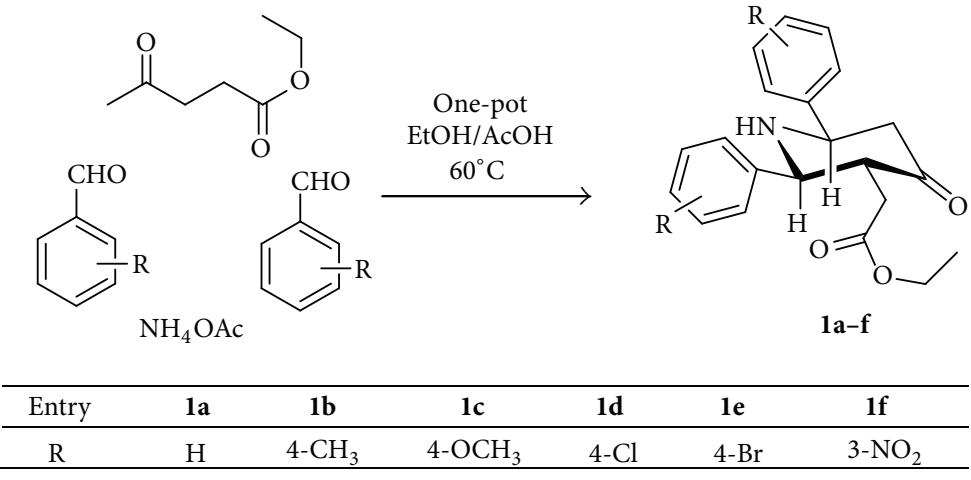

Scheme 1: Synthesis of piperidin-4-one derivatives. Reagents and conditions: methanol-acetic acid (70 mol\%), $60^{\circ} \mathrm{C}, 2 \mathrm{~h}$.

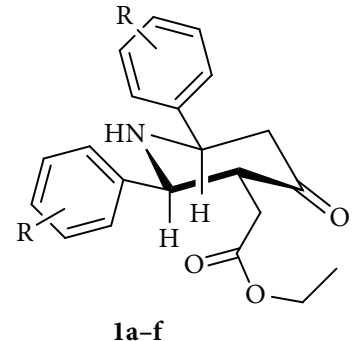

1a-f

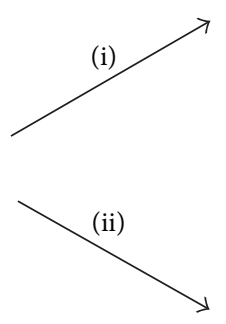

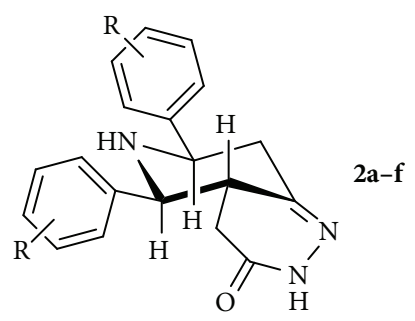

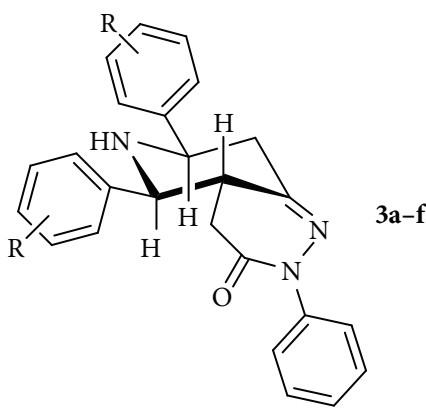

\begin{tabular}{lcccccc}
\hline Entry & $\mathbf{2 a} / \mathbf{3 a}$ & $\mathbf{2 b} / \mathbf{3 b}$ & $\mathbf{2 c} / \mathbf{3 c}$ & $\mathbf{2 d} / \mathbf{3 d}$ & $\mathbf{2 e} / \mathbf{3 e}$ & $\mathbf{2 f} / \mathbf{3 f}$ \\
\hline $\mathrm{R}$ & $\mathrm{H}$ & $4-\mathrm{CH}_{3}$ & $4-\mathrm{OCH}_{3}$ & $4-\mathrm{Cl}$ & $4-\mathrm{Br}$ & $3-\mathrm{NO}_{2}$ \\
\hline
\end{tabular}

Scheme 2: Synthesis of pyridopyridazin-3(2H)-one derivatives. Reagents and conditions: (i) $\mathrm{N}_{2} \mathrm{H}_{4} \cdot \mathrm{H}_{2} \mathrm{O}$, ethanol, reflux, $17 \mathrm{~h}$; (ii) $\mathrm{PhNHNH}$, dry toluene, TFA (20 mol\%), reflux, 2 days.
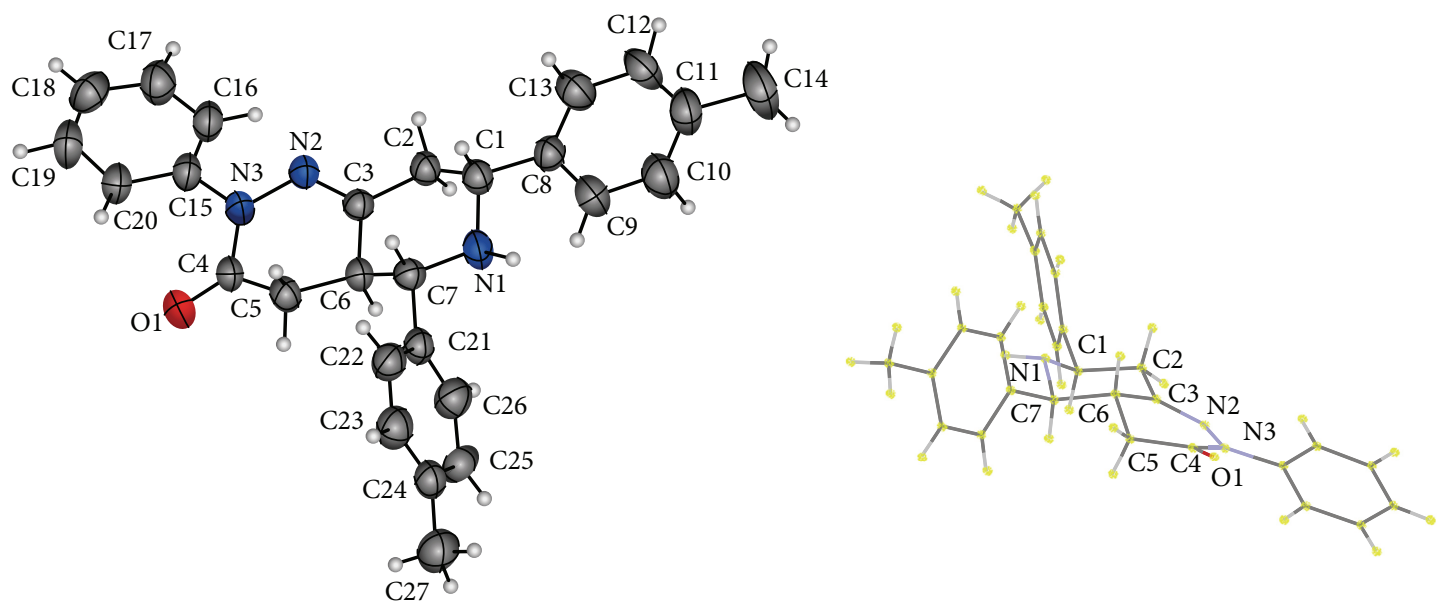

FIGURE 1: ORTEP diagram of $\mathbf{3} \mathbf{b}$ derived from single crystal XRD analysis. 
TABLE 1: Inhibitory effects of compounds $\mathbf{2 a}-\mathbf{f}, \mathbf{3 a}-\mathbf{f}$ on viability of breast cancer cells.

\begin{tabular}{lcc}
\hline Compounds & $\mathrm{R}$ & MCF-7 $\mathrm{IC}_{50}{ }^{\mathrm{a}}(\mu \mathrm{M})$ \\
\hline 2a & $\mathrm{H}$ & $475.4 \pm 27.8$ \\
2b & $\mathrm{CH}_{3}$ & $498.4 \pm 30.0$ \\
2c & $\mathrm{OCH}_{3}$ & $344.1 \pm 59.4$ \\
2d & $\mathrm{Cl}$ & $61.3 \pm 5.8$ \\
2e & $\mathrm{Br}$ & $78.9 \pm 14.7$ \\
2f & $3-\mathrm{NO}_{2}$ & $141.0 \pm 22.5$ \\
3a & $\mathrm{H}$ & $268.7 \pm 15.4$ \\
3b & $\mathrm{CH}_{3}$ & $219.0 \pm 11.0$ \\
3c & $\mathrm{OCH}_{3}$ & $248.1 \pm 17.2$ \\
3d & $\mathrm{Cl}$ & $364.1 \pm 20.2$ \\
3e & $\mathrm{Br}$ & $810.0 \pm 35.7$ \\
3f & $3-\mathrm{NO}_{2}$ & $473.7 \pm 26.1$ \\
Doxorubicin $^{\mathrm{b}}$ & - & $2.007 \pm 0.05$ \\
\hline
\end{tabular}

${ }^{a}$ Inhibitory concentration $\left(\mathrm{IC}_{50}, \mu \mathrm{M}\right)$ as obtained from MTT assay. All the values represent the average of three experimental samples. The cells were incubated for a period of $48 \mathrm{~h}$.

${ }^{\mathrm{b}}$ Doxorubicin was used as the standard drug.

incorporated derivatives $\mathbf{2 d - f}$ and not the phenylhydrazine incorporated derivatives $\mathbf{3 d} \mathbf{d}-\mathbf{f}$ show the high activity against MCF-7 breast cancer cell line. Thus, the aryl ring at position 3 seems unimportant and, in fact, reduces the activity against MCF-7 cells.

\section{Conclusion}

In conclusion, three efficient methods for the syntheses of different derivatives of ethyl 4-oxo-2,6-diarylpiperidin-3yl acetate, 4,4a,5,6,7,8-hexahydro-5,7-diarylpyrido[4,3-c]pyridazin-3(2H)-one, and 4,4a,5,6,7,8-hexahydro-2,5,7-triarylpyrido[4,3-c]pyridazin-3(2H)-one are reported. The yields of ethyl 4-oxo-2,6-diarylpiperidin-3yl acetates (1a-f) are improved significantly when glacial acetic acid is used as the catalyst. The requirement of a strongly acidic 20\% TFA/ toluene medium for the formation of cyclic products $\mathbf{3 a - f}$ is also demonstrated. Further, the inhibitory activity of the test compounds against MCF-7 human breast adenocarcinoma cells is provided. A comparative account explaining the importance of functional groups for the higher levels of activity shown by $\mathbf{2 d}, \mathbf{2 e}$, and $\mathbf{2} \mathbf{f}$ is also presented.

\section{Experimental Section}

4.1. General. All the chemicals used in this study were purchased from Sigma Aldrich without further purification. Melting points were determined in open capillary tubes and are uncorrected. IR spectra were taken as $\mathrm{KBr}$ pellets for solids on a Perkin Elmer Spectrum RXI FT-IR. ${ }^{1} \mathrm{H}$ NMR $(500 \mathrm{MHz})$ and ${ }^{13} \mathrm{C}$ NMR $(125 \mathrm{MHz})$ spectra were recorded in $\mathrm{CDCl}_{3}$ solutions with TMS as an internal standard on a JEOL instrument and Brucker Avance instrument. Mass spectra were recorded on a Thermo Finnigan LCQ Advantage MAX 6000 ESI spectrometer. Column chromatography was performed using neutral aluminium oxide, 150-200 mesh, and eluent, $7 \%$ ethyl acetate in hexane.

4.2. General Procedure for the Synthesis of 4-Oxo-2,6diphenylpiperidin-3-yl-acetate Derivatives (1a-f). 4-Oxo2,6-diphenylpiperidin-3-yl-acetate (1a) was synthesized as described elsewhere[23] with a slight modification. Methanol was used as the solvent and glacial acetic acid (70 mol\%) was used as the catalyst. In a typical experiment, ammonium acetate $(3.85 \mathrm{~g}, 50 \mathrm{mmol})$ was dissolved in methanol $(60 \mathrm{~mL})$ and then benzaldehyde $(9.0 \mathrm{~mL}, 100 \mathrm{mmol})$ was added and warmed over a water-bath for $5 \mathrm{~min}$. Ethyl levulinate $(7.0 \mathrm{~mL}$, $50 \mathrm{mmol})$ and glacial acetic acid $(2.0 \mathrm{~mL}, 35 \mathrm{mmol})$ were added and heated at $\sim 60^{\circ} \mathrm{C}$ over a water-bath for $2 \mathrm{~h}$ and then left aside at room temperature. The pale-yellow precipitate formed was crystallized from methanol.

4.3. Synthesis of 4,4a,5,6,7,8-Hexahydro-5,7-diphenylpyrido [4,3-c]pyridazin-3(2H)-ones $(\mathbf{2 a - 2 f )}$. To a solution of appropriate ethyl 4-oxo-2,6-diphenylpiperidin-3-yl-acetates (337 $\mathrm{mg}, 1.0 \mathrm{mmol}$ ) in ethanol was added hydrazine hydrate $(0.053 \mathrm{~mL}, 1.2 \mathrm{mmol})$ and the resultant solution was refluxed for $17 \mathrm{~h}$. The solvent was evaporated and the residue obtained was extracted with chloroform $(3 \times 20 \mathrm{~mL})$. The combined organic layer was dried over sodium sulphate, concentrated under reduced pressure and the solid obtained was purified by crystallization using methanol.

4.3.1. 4,4a,5,6,7,8-Hexahydro-5,7-diphenylpyrido[4,3-c]pyridazin-3(2H)-one (2a). Specifications are as follows: colourless solid; mp 153- $154^{\circ} \mathrm{C}$; yield $82 \%$; IR $\left(\mathrm{KBr} \mathrm{cm}^{-1}\right)$ : 3371, 3281, 3025, 2925, 2848, 1685, 1514, 1416, 1334, 1106, 1038, 823, 759; ${ }^{1} \mathrm{H}$ NMR $\left(500 \mathrm{MHz}, \mathrm{CDCl}_{3}\right): \delta \mathrm{ppm} 2.10$ (bs, $1 \mathrm{H}$, amine $\mathrm{NH}), 2.17-2.28(\mathrm{~m}, 2 \mathrm{H}), 2.51-2.56(\mathrm{~m}, 1 \mathrm{H}), 2.71-2.76(\mathrm{~m}, 2 \mathrm{H}$ merged), $3.63(\mathrm{~d}, 1 \mathrm{H}, J=9.7 \mathrm{~Hz}), 3.9(\mathrm{~d}, 1 \mathrm{H}, J=11.4 \mathrm{~Hz})$, 7.26-7.35 (m, 6H Ar protons), 7.41-7.44 (m, 4H Ar protons), 8.77 (bs, $1 \mathrm{H}$, amide $\mathrm{NH}) ;{ }^{13} \mathrm{C} \mathrm{NMR}\left(125 \mathrm{MHz} \mathrm{CDCl}_{3}\right): \delta \mathrm{ppm}$ 29.7, 40.5, 41.7, 60.9, 68.3, 126.7, 127.6, 127.9, 128.6, 128.7, 128.9, $140.5,142.9,152.8,166.3 ; \mathrm{MS} m / z=306[\mathrm{MH}]^{+}$.

4.3.2. 4,4a,5,6,7,8-Hexahydro-5,7-bis(4-methylphenyl)pyrido [4,3-c]pyridazin-3(2H)-one (2b). Specifications are as follows: colourless solid; mp $183-185^{\circ} \mathrm{C}$; yield $72 \%$; IR $(\mathrm{KBr}$ $\left.\mathrm{cm}^{-1}\right)$ : 3303, 3210, 3087, 2944, 2831, 1665, 1611, 1511, 1458, 1303, $1367,1241,1175,1107,1031,834 ;{ }^{1} \mathrm{H}$ NMR $\left(500 \mathrm{MHz}, \mathrm{CDCl}_{3}\right)$ : $\delta$ ppm 2.07 (bs, $1 \mathrm{H}$, amine $\mathrm{NH}), 2.18-2.34(\mathrm{~m}, 2 \mathrm{H}), 2.36(\mathrm{~s}$, $3 \mathrm{H}), 2.37$ (s, 3H), $2.54(\mathrm{dd}, 1 \mathrm{H}, J=15,12 \mathrm{~Hz}), 2.72-2.78(\mathrm{~m}$, $2 \mathrm{H}$ merged), $3.62(\mathrm{~d}, 1 \mathrm{H}, J=9.5 \mathrm{~Hz}), 3.96(\mathrm{dd}, 1 \mathrm{H}, J=12$, $3 \mathrm{~Hz}$ ), 7.18 (d, $4 \mathrm{H}, J=6.5 \mathrm{~Hz}$ Ar protons), 7.32-7.35 (m, $4 \mathrm{H}$ Ar protons), 8.66 (bs, $1 \mathrm{H}$, amide $\mathrm{NH}) ;{ }^{13} \mathrm{C} \mathrm{NMR}(125 \mathrm{MHz}$, $\left.\mathrm{CDCl}_{3}\right): \delta$ ppm 21.12, 21.14, 29.6, 40.4, 41.7, 60.6, 68.0, 126.4, $127.4,129.3,129.4,137.51,137.55,138.2,140.0,152.9,166.2$; MS $m / z=334[\mathrm{MH}]^{+}$.

4.3.3. 4,4a,5,6,7,8-Hexahydro-5,7-bis(4-methoxyphenyl)pyrido [4,3-c]pyridazin-3(2H)-one $(2 \mathrm{c})$. Specifications are as follows: colourless solid; mp $188-190^{\circ} \mathrm{C}$; yield $76.7 \%$; IR ( $\mathrm{KBr}$ $\left.\mathrm{cm}^{-1}\right)$ : 3318, 3222, 3090, 2920, 2849, 1664, 1439, 1414, 1370, 
1305, 1230, 1174, 1091, 1014, 828; ${ }^{1} \mathrm{H}$ NMR (500 MHz, $\left.\mathrm{CDCl}_{3}\right)$ : $\delta$ ppm 2.04 (bs, $1 \mathrm{H}$, amine $\mathrm{NH}$ ), 2.17-2.33 (m, 2H), 2.52 (dd, $1 \mathrm{H}, J=15.5,11.5 \mathrm{~Hz}), 2.69-2.75(\mathrm{~m}, 2 \mathrm{H}$ merged), $3.59(\mathrm{~d}$, $1 \mathrm{H}, J=9.5 \mathrm{~Hz}), 3.81(\mathrm{~s}, 3 \mathrm{H}), 3.82(\mathrm{~s}, 3 \mathrm{H}), 3.93(\mathrm{dd}, 1 \mathrm{H}, J=$ $11.5,3 \mathrm{~Hz}), 6.88-6.90$ (m, $4 \mathrm{H}$ Ar protons), 7.34-7.38 (m, $4 \mathrm{H}$ Ar protons), 8.66 (bs, $1 \mathrm{H}$, amide $\mathrm{NH}) ;{ }^{13} \mathrm{C}$ NMR $(125 \mathrm{MHz}$, $\left.\mathrm{CDCl}_{3}\right): \delta$ ppm 29.6, 40.6, 41.7, 55.30, 55.34, 60.3, 67.7, 114.0, $114.1,127.6,128.6,132.6,135.1,152.9,159.1,159.6,166.2$; MS $m / z=366[\mathrm{MH}]^{+}$.

4.3.4. 4,4a,5,6,7,8-Hexahydro-5,7-bis(4-chlorophenyl)pyrido[4, 3-c]pyridazin-3(2H)-one (2d). Specifications are as follows: colourless solid, mp $185-187^{\circ} \mathrm{C}$, yield $79 \%$, IR $\left(\mathrm{KBr} \mathrm{cm}^{-1}\right)$ : 3425, 3322, 3225, 3086, 2931, 2367, 2339, 1663, 1525, 1478, 1353, 1211, 1166; ${ }^{1} \mathrm{H}$ NMR $\left(500 \mathrm{MHz}, \mathrm{CDCl}_{3}\right): \delta$ ppm 2.07 (bs, $1 \mathrm{H}$, amine $\mathrm{NH}), 2.16-2.30(\mathrm{~m}, 2 \mathrm{H}), 2.48(\mathrm{dd}, 1 \mathrm{H}, J=15,11.5 \mathrm{~Hz})$, 2.67-2.75 (m, 2H merged), 3.63 (d, $1 \mathrm{H}, J=9.5 \mathrm{~Hz}$ ), 3.96 (dd, $1 \mathrm{H}, J=11.5,3 \mathrm{~Hz}$ ), $7.32-7.39$ (m, $8 \mathrm{H}$ Ar protons), 8.75 (bs, $1 \mathrm{H}$, amide $\mathrm{NH}) ;{ }^{13} \mathrm{C}$ NMR $\left(125 \mathrm{MHz} \mathrm{CDCl}_{3}\right): \delta$ ppm 29.5, 40.4, 41.6, 60.1, 67.4, 127.9, 128.9, 129.0, 133.6, 134.4, 138.8, 141.1, 151.6, 165.8; MS $m / z=374[\mathrm{MH}]^{+}$.

4.3.5. 4,4a,5,6,7,8-Hexahydro-5,7-bis(4-bromophenyl)pyrido[4, 3-c]pyridazin-3(2H)-one (2e). Specifications are as follows: brown solid, $\mathrm{mp} 133-135^{\circ} \mathrm{C}$, yield $75 \%$, IR $\left(\mathrm{KBr} \mathrm{cm}^{-1}\right)$ : 3236 , 3097, 2922, 2854, 1674, 1485, 1439, 1412, 1345, 1305, 1230, 1102, 1070, 1008, 825; ${ }^{1} \mathrm{H}$ NMR (500 MHz, $\mathrm{CDCl}_{3}$ ): $\delta$ ppm 2.07 (bs, $1 \mathrm{H}$, amine $\mathrm{NH}), 2.17-2.31(\mathrm{~m}, 2 \mathrm{H}), 2.48(\mathrm{dd}, 1 \mathrm{H}, J=15$, $12 \mathrm{~Hz}$ ), 2.67-2.75 (m, 2H merged), $3.62(\mathrm{~d}, 1 \mathrm{H}, \mathrm{J}=10 \mathrm{~Hz}), 3.95$ (dd, $1 \mathrm{H}, J=11.5,3 \mathrm{~Hz}), 7.31$ (d, $4 \mathrm{H}, J=8.5 \mathrm{~Hz}$ Ar protons), $7.48-7.53$ (m, $4 \mathrm{H}$ Ar protons), 8.67 (bs, $1 \mathrm{H}$, amide $\mathrm{NH}$ ); ${ }^{13} \mathrm{C}$ NMR $\left(125 \mathrm{MHz}, \mathrm{CDCl}_{3}\right): \delta$ ppm 29.5, 40.4, 41.5, 60.2, 67.5, 121.7, 122.5, 128.2, 129.2, 131.8, 132.0, 139.2, 141.6, 151.5, 165.7; MS $m / z=463[\mathrm{MH}]^{+}$.

4.3.6. 4,4a,5,6,7,8-Hexahydro-5,7-bis(3-nitrophenyl)pyrido[4, 3-c]pyridazin-3(2H)-one (2f). Specifications are as follows: pale brown solid, $\mathrm{mp} 248-250^{\circ} \mathrm{C}$, yield $69 \%$, IR $\left(\mathrm{KBr} \mathrm{cm}^{-1}\right)$ : 3417, 3322, 3227, 3091, 2926, 2853, 1665, 1523, 1353, 1453, 808, 735,$688 ;{ }^{1} \mathrm{H}$ NMR $\left(500 \mathrm{MHz}, \mathrm{CDCl}_{3}\right): \delta \mathrm{ppm} 1.89(\mathrm{dd}, 1 \mathrm{H}, J$ $=9,17.5 \mathrm{~Hz}), 2.32(\mathrm{dd}, 1 \mathrm{H}, J=13.5,17.5 \mathrm{~Hz}), 2.42-2.48(\mathrm{~m}, 1 \mathrm{H})$, $2.70(\mathrm{dd}, 1 \mathrm{H}, J=14.5,3 \mathrm{~Hz}), 2.81-2.87(\mathrm{~m}, 1 \mathrm{H}), 3.90(\mathrm{~d}, 1 \mathrm{H}, J=$ $10 \mathrm{~Hz}), 4.11(\mathrm{~d}, 1 \mathrm{H}, J=10.5 \mathrm{~Hz}), 7.64-7.70$ ( $\mathrm{m}, 2 \mathrm{H}$ Ar protons), 7.93-7.97 (m, $2 \mathrm{H}$ Ar protons), 8.16 (2 set of dd, $2 \mathrm{H}, J=8$, $1.5 \mathrm{~Hz} 8.5,2 \mathrm{~Hz}$ Ar protons), 8.34-8.39 (m, 2H Ar protons), 10.6 (bs, $1 \mathrm{H}$, amide $\mathrm{NH}) ;{ }^{13} \mathrm{C} \mathrm{NMR}\left(125 \mathrm{MHz}, \mathrm{CDCl}_{3}\right): \delta \mathrm{ppm}$ 29.7, 39.3, 40.8, 59.0, 66.0, 121.8, 122.6, 123.1, 123.2, 130.3, 134.2, $135.2,144.0,146.1,148.2,148.3,150.6,165.8$. Ms $m / z=395.34$ $\left[\mathrm{M}^{+}\right]$.

4.4. Synthesis of 2-Phenyl-5,7-diarylpyrido[4,3-c]pyridazin3(2H)-one Derivatives $(\mathbf{3 a}-\mathbf{3 f})$. A mixture of ethyl 4-oxo-2,6diphenylpiperidin-3-yl-acetates (337 $\mathrm{mg}, 1.0 \mathrm{mmol})$ in dry toluene were added to phenyl hydrazine $(0.118 \mathrm{~mL}, 1.2 \mathrm{mmol})$ and trifluoroacetic acid $(0.0155 \mathrm{~mL}, 0.20 \mathrm{mmol})$, and the solution was refluxed under nitrogen atmosphere for 2 days. After the reaction was complete the solvent was evaporated under reduced pressure, and the residue obtained was extracted with chloroform $(3 \times 20 \mathrm{~mL})$. The combined organic layer was dried over anhydrous sodium sulfate and concentrated under reduced pressure and the residue obtained was purified using column chromatography (neutral aluminium oxide, 150-200 mesh; eluent: $7 \%$ ethyl acetate in hexane).

4.4.1. 4,4a,5,6,7,8-Hexahydro-2,5,7-triphenylpyrido[4,3-c]pyridazin-3(2H)-one (3a). Specifications are as follows: pale brown solid; mp 140-143 ${ }^{\circ} \mathrm{C}$; yield 79\%; IR $\left(\mathrm{KBr} \mathrm{cm}^{-1}\right)$ : 3304 , 3030, 2960, 2887, 2806, 1680, 1595, 1492, 1454, 1326, 1305, $1227,1160,1107,752 ;{ }^{1} \mathrm{H}$ NMR $\left(500 \mathrm{MHz}, \mathrm{CDCl}_{3}\right): \delta \mathrm{ppm} 2.18$ (bs, $1 \mathrm{H}$, amine $\mathrm{NH}), 2.43-2.54(\mathrm{~m}, 2 \mathrm{H}), 2.66(\mathrm{dd}, 1 \mathrm{H}, J=15$, $11.5 \mathrm{~Hz}$ ), 2.90-2.96 (m, $2 \mathrm{H}$ merged), 3.73 (d, $1 \mathrm{H}, J=9.5 \mathrm{~Hz}$ ), 4.07 (dd, $1 \mathrm{H}, J=11.5,2.5 \mathrm{~Hz}$ ), 7.27-7.33 (m, $2 \mathrm{H}$ Ar protons), 7.34-7.44 (m, 7H Ar protons), 7.48-7.52 (m, 6H Ar protons); ${ }^{13} \mathrm{C} \mathrm{NMR}\left(125 \mathrm{MHz}, \mathrm{CDCl}_{3}\right): \delta \mathrm{ppm} 31.3,41.0,41.7,60.8,68.4$, $124.9,126.5,126.7,127.6,127.9,128.6,128.7,128.8,140.6,140.9$, $142.9,153.6,164.3 ; \mathrm{MS} m / z=382[\mathrm{MH}]^{+}$.

4.4.2. 4,4a,5,6,7,8-Hexahydro-5,7-bis(4-methylphenyl)-2-phenylpyrido[4,3-c]pyridazin-3(2H)-one (3b). Specifications are as follows: pale brown solid; $\mathrm{mp} 166-167^{\circ} \mathrm{C}$; yield $72 \%$; IR ( $\mathrm{KBr}$ $\left.\mathrm{cm}^{-1}\right)$ : 3320, 3021, 2919, 2878, 2815, 1688, 1594, 1492 1321, 1299, $1228,1155,1105,821,756 ;{ }^{1} \mathrm{H}$ NMR $\left(500 \mathrm{MHz}, \mathrm{CDCl}_{3}\right): \delta \mathrm{ppm}$ 2.08 (bs, 1H, amine NH), 2.38 (s, 3H), 2.39 (s, 3H), 2.41$2.51(\mathrm{~m}, 2 \mathrm{H}), 2.64(\mathrm{dd}, 1 \mathrm{H}, J=15,12 \mathrm{~Hz}), 2.87-2.91(\mathrm{~m}, 2 \mathrm{H}$ merged), 3.69 (d, $1 \mathrm{H}, J=9.5 \mathrm{~Hz}), 4.03(\mathrm{dd}, 1 \mathrm{H}, J=11.5,3 \mathrm{~Hz})$, 7.19-7.22 (m, 4H, Ar protons), 7.28-7.29 ( $\mathrm{m}, 1 \mathrm{H}$, Ar protons), 7.36-7.38 (m, 4H, Ar protons), 7.40-7.43 ( $\mathrm{m}, 2 \mathrm{H}$, Ar protons), 7.51 (d, $2 \mathrm{H}, J=8 \mathrm{~Hz}$ Ar protons); ${ }^{13} \mathrm{C} \mathrm{NMR}\left(125 \mathrm{MHz}, \mathrm{CDCl}_{3}\right)$ : $\delta$ ppm 21.14, 21.16, 31.3, 41.0, 41.8, 60.6, 68.1, 124.9, 126.4, 126.6, $127.4,128.6,129.3,129.5,137.5,137.6,140.0,141.0,153.9,164.4$; MS $m / z=410[\mathrm{MH}]^{+}$.

4.4.3. 4,4a,5,6,7,8-Hexahydro-5,7-bis(4-methoxyphenyl)-2-phenylpyrido[4,3-c]pyridazin-3(2H)-one (3c). Specifications are as follows: pink solid, $\mathrm{mp} 128-130^{\circ} \mathrm{C}$; yield $74 \%$; IR ( $\mathrm{KBr}$ $\mathrm{cm}^{-1}$ ): 3415, 3306, 2954, 2832, 1682, 1606, 1506, 1449, 1302, $1242,1175,1105,927,833,752 ;{ }^{1} \mathrm{H}$ NMR $\left(500 \mathrm{MHz}, \mathrm{CDCl}_{3}\right)$ : $\delta$ ppm 1.99 (bs, $1 \mathrm{H}$, amine $\mathrm{NH}$ ), 2.39-2.54 (m, 2H), 2.62 (dd, $1 \mathrm{H}, J=14.5,12 \mathrm{~Hz}), 2.84-2.88(\mathrm{~m}, 2 \mathrm{H}$ merged $), 3.66(\mathrm{~d}, 1 \mathrm{H}$, $J=9.5 \mathrm{~Hz}), 3.82(\mathrm{~s}, 3 \mathrm{H}), 3.84(\mathrm{~s}, 3 \mathrm{H}), 4.00(\mathrm{dd}, 1 \mathrm{H}, J=12$, $3 \mathrm{~Hz}$ ), 6.90-6.93 (m, 4H Ar protons), 7.27-7.28 (m, 1H, Ar proton), $7.38-7.43$ (m, 6H, Ar protons), 7.50 (d, $2 \mathrm{H}, J=8 \mathrm{~Hz}$ Ar protons); ${ }^{13} \mathrm{C} \mathrm{NMR} \mathrm{(125} \mathrm{MHz}, \mathrm{CDCl}_{3}$ ): $\delta \mathrm{ppm}$ 31.1, 41.0, 41.7, 55.20, 55.27, 60.1, 67.2, 113.9, 114.0, 124.9, 126.6, 127.5, 128.5, $132.6,135.0,140.8,153.9,159.0,159.6,164.3$; MS $m / z=442$ $[\mathrm{MH}]^{+}$.

4.4.4. 4,4a,5, 6,7,8-Hexahydro-5,7-bis(4-chlorophenyl)-2-phenylpyrido[4,3-c]pyridazin-3(2H)-one (3d). Specifications are as follows: pale brown solid; $\mathrm{mp} 113-115^{\circ} \mathrm{C}$; yield $77 \%$; IR $\left(\mathrm{KBr} \mathrm{cm}^{-1}\right): 3315,3061,2853,2810,1676,1593,1491,1442,1413$, $1359,1329,1304,1227,1202,1158,1093,1014,830 ;{ }^{1} \mathrm{H}$ NMR $\left(500 \mathrm{MHz}, \mathrm{CDCl}_{3}\right): \delta$ ppm 2.07 (bs, $1 \mathrm{H}$, amine $\mathrm{NH}$ ), 2.39$2.51(\mathrm{~m}, 2 \mathrm{H}), 2.57(\mathrm{dd}, 1 \mathrm{H}, J=15,12 \mathrm{~Hz}), 2.81-2.88(\mathrm{~m}, 2 \mathrm{H}$ merged), 3.69 (d, $1 \mathrm{H}, J=10 \mathrm{~Hz}), 4.02(\mathrm{dd}, 1 \mathrm{H}, J=12,3 \mathrm{~Hz})$, 7.27-7.29 (m, $1 \mathrm{H}$ Ar protons), 7.34-7.44 (m, 10H Ar protons), 
7.48 (d, $2 \mathrm{H}, J=8.5 \mathrm{~Hz}$ Ar protons); ${ }^{13} \mathrm{C}$ NMR $(125 \mathrm{MHz}$, $\left.\mathrm{CDCl}_{3}\right): \delta$ ppm 31.3, 40.9, 41.7, 60.1, 67.5, 124.9, 126.8, 127.9, $128.6,128.9,128.9,129.1,133.6,134.4,138.9,140.8,141.2,152.6$, 163.9; $\mathrm{MS} m / z=450[\mathrm{MH}]^{+}$.

4.4.5. 4,4a,5,6,7,8-Hexahydro-5,7-bis(4-bromophenyl)-2-phenylpyrido[4,3-c]pyridazin-3(2H)-one (3e). Specifications are as follows: pale brown solid; $\mathrm{mp} 101-102^{\circ} \mathrm{C}$; yield $71.6 \%$; IR $\left(\mathrm{KBr} \mathrm{cm}^{-1}\right): 3300,3041,2923,2814,1679,1593,1488,1410,1328$, 1305, 1227, 1201, 1159, 1106, 1069, 827; ${ }^{1} \mathrm{H}$ NMR $(500 \mathrm{MHz}$, $\mathrm{CDCl}_{3}$ ): $\delta$ ppm 2.16 (bs, $1 \mathrm{H}$, amine $\left.\mathrm{NH}\right), 2.36-2.48(\mathrm{~m}, 2 \mathrm{H})$, $2.53(\mathrm{dd}, 1 \mathrm{H}, J=15.5,11.5 \mathrm{~Hz}$ ), 2.78-2.84 (m, 2H merged), 3.65 $(\mathrm{d}, 1 \mathrm{H}, J=10 \mathrm{~Hz}), 3.98(\mathrm{dd}, 1 \mathrm{H}, J=12.5,3 \mathrm{~Hz}), 7.23-7.26(\mathrm{~m}, 1 \mathrm{H}$ Ar protons), 7.31-7.39 (m, 6H Ar protons), 7.43-7.52 (m, 6H Ar protons); $\left.{ }^{13} \mathrm{C} \mathrm{NMR} \mathrm{(125} \mathrm{MHz,} \mathrm{CDCl}_{3}\right): \delta$ ppm 31.2, 41.0, 41.7, 60.2, 67.6, 121.8, 122.6, 125.0, 126.9, 128.3, 131.9, 132.1, 139.4, $140.8,141.7,152.6,164.0 ; \mathrm{MS} m / z=539[\mathrm{MH}]^{+}$.

4.4.6. 4,4a,5,6,7,8-Hexahydro-5,7-bis(3-nitrophenyl)-2-phenyIpyrido[4,3-c]pyridazin-3(2H)-one (3f). Specifications are as follows: brown solid; m.p 190-191 ${ }^{\circ} \mathrm{C}$; yield 67\%; IR ( $\mathrm{KBr}$ $\left.\mathrm{cm}^{-1}\right): 3418,3085,2922,2852,1679,1596,1522,1345,1103$, 908, 810; ${ }^{1} \mathrm{H}$ NMR $\left(500 \mathrm{MHz}, \mathrm{CDCl}_{3}\right): \delta$ ppm 2.04 (bs, amine $\mathrm{NH}), 2.42-2.52$ (m, 2H), 2.64 (dd, $1 \mathrm{H}, J=11.5,15.5 \mathrm{~Hz}), 2.91-$ 2.97 (m, $2 \mathrm{H}$ merged), 3.88 (d, 1H, $J=10 \mathrm{~Hz}$ ), 4.19 (dd, $1 \mathrm{H}, J$ $=12.5,3 \mathrm{~Hz}), 7.24-7.27$ (m, $1 \mathrm{H}$ Ar protons), 7.35-7.40 (m, $2 \mathrm{H}$ Ar protons), 7.44 (d, 2H, $J=8 \mathrm{~Hz}$ Ar protons), 7.55-7.62 (m, 2H Ar protons), 7.81-7.85 (m, $2 \mathrm{H}$ Ar protons), 8.18 (d, 1H, $J=$ $8 \mathrm{~Hz}$ Ar protons), 8.23 (d, $1 \mathrm{H}, J=9 \mathrm{~Hz}$ Ar protons), 8.35-8.39 (m, $2 \mathrm{H}$ Ar protons); ${ }^{13} \mathrm{C}$ NMR (125 MHz, $\mathrm{CDCl}_{3}$ ): $\delta \mathrm{ppm} 31.1$, 40.8, 41.4, 60.1, 67.3, 121.8, 122.7, 123.3, 123.9, 125.0, 127.0, 128.8, $130.0,130.2,132.9,133.9,140.7,142.3,144.4,148.6,148.7,151.3$, 163.5. Ms $m / z=471.42[\mathrm{MH}]^{+}$.

4.5. Pharmacological Study: Chemicals and Reagents. Human breast adenocarcinoma (MCF-7) cell culture was procured from the National Centre for Cell Sciences (NCCS), Pune, India. The cells were grown in Dulbecco's Modified Eagle Medium (DMEM) supplemented with $10 \%$ heat inactivated fetal bovine serum (FBS), penicillin $(100 \mathrm{IU} / \mathrm{mL})$, streptomycin $(100 \mu \mathrm{g} / \mathrm{mL})$, and amphotericin-B $(5 \mu \mathrm{g} / \mathrm{mL})$ in a humidified atmosphere of $5 \% \mathrm{CO}_{2}$ at $37^{\circ} \mathrm{C}$ until confluent. The cells were trypsinized with TPVG solution ( $0.2 \%$ trypsin, $0.02 \%$ EDTA, $0.05 \%$ glucose in PBS). The stock cultures were grown in $25 \mathrm{~cm}^{2}$ flat bottles and the studies were carried out in 96-well microtiter plates.

4.5.1. Cell Culture and In Vitro Cytotoxicity Assay. Cells were plated in 96-well flat bottom microtiter plate at a density of 1 $\times 10^{4}$ cells per well and cultured for $24 \mathrm{~h}$ at $37^{\circ} \mathrm{C}$ in $5 \% \mathrm{CO}_{2}$ atmosphere to allow cell adhesion. After $24 \mathrm{~h}$, when partial monolayer was formed, medium was removed and cells were treated with different concentrations of standard drug (doxorubicin) and test compounds. Microscopic examination was carried out and observations recorded every $24 \mathrm{~h}$. After the treatment, the solutions in the wells were discarded and $50 \mu \mathrm{L}$ of the freshly prepared MTT solution ( $2 \mathrm{mg} / \mathrm{mL}$ PBS) was added to each well. The plates were gently shaken and incubated for $3 \mathrm{~h}$ at $37^{\circ} \mathrm{C}$ in $5 \% \mathrm{CO}_{2}$ atmosphere. After $3 \mathrm{~h}$, the supernatant was removed and the formazan crystals formed in the cells were solubilized by adding isopropanol $(50 \mu \mathrm{L})$. Finally, the amount of formazan formed in different wells was measured from the absorbance at $540 \mathrm{~nm}$ using a microplate reader (Bio-Tek, EL X-800 MS). The concentration of drug required to kill $50 \%$ of cells in exponentially growing cultures after a $48 \mathrm{~h}$ exposure to the drug $\left(\mathrm{IC}_{50}\right.$ values) was calculated from the plot of $\mathrm{A}_{540}$ versus concentration of test sample. The cytotoxicity of the complex was measured from the spectrophotometric data by means of this equation: \% cell cytotoxicity $=\left[\mathrm{Abs}_{\text {control }}-\mathrm{Abs}_{\text {test }} / \mathrm{Abs}_{\text {control }}\right] \times 100$.

\section{Conflict of Interests}

The authors declare that there is no conflict of interests regarding the publication of this paper.

\section{Acknowledgments}

One of the authors, Periasamy Selvakumar, thanks the Council of Scientific and Industrial Research, New Delhi, India, for the research fellowship. The authors would like to acknowledge the Department of Chemistry, IIT-Madras, India, for single crystal XRD analysis and the Department of Pharmaceutical Biotechnology, Manipal College of Pharmaceutical Sciences, Manipal, Karnataka, India, for MTT Assay. Financial support from CSC0201 project of CSIR is acknowledged.

\section{References}

[1] T. Taniguchi and K. Ogasawara, "A diastereocontrolled synthesis of (+)-febrifugine: a potent antimalarial piperidine alkaloid," Organic Letters, vol. 2, no. 20, pp. 3193-3195, 2000.

[2] Y. Takeuchi, K. Azuma, K. Takakura, H. Abe, and T. Harayama, "Asymmetric synthesis of febrifugine and isofebrifugine using yeast reduction," Chemical Communications, no. 17, pp. 16431644, 2000.

[3] H. I. El-Subbagh, S. M. Abu-Zaid, M. A. Mahran, F. A. Badria, and A. M. Al-Obaid, "Synthesis and biological evaluation of certain $\alpha, \beta$-unsaturated ketones and their corresponding fused pyridines as antiviral and cytotoxic agents," Journal of Medicinal Chemistry, vol. 43, no. 15, pp. 2915-2921, 2000.

[4] B. R. Jerom and K. H. Spencer, "Preparation and testing of 4(heterocyclylacylamino)piperidines as narcotic antagonists and analgesics," European Patent Applications EP 277794, 1988.

[5] R. Venkatesa Perumal, M. Adiraj, and P. Shanmuga Pandiyan, "Synthesis, analgesic and anti inflammatory evaluation of substituted 4-piperidones," Indian Drugs, vol. 38, no. 3, pp. 156-159, 2001.

[6] R. E. Hagenbach and H. Gysin, "Über einige heterozyklische Thiosemicarbazone," Experientia, vol. 8, no. 5, pp. 184-185, 1952.

[7] I. G. Mobio, A. T. Soldatenkov, V. O. Fedorov et al., "Synthesis and physiological activity of 2,3,6-triaryl-4-oxo (hydroxy, oximino, amino) piperidine," Khimiko Farmatsevticheskii Zhurnal, vol. 23, no. 4, pp. 421-427, 1989.

[8] A. R. Katritzky and W.-Q. Fan, "A novel and versatile synthesis of 1-alkyl-, 1-aryl-, 1-(alkylamino)-, or 1-amido-substituted and 
of 1,2,6-trisubstituted piperidines from glutaraldehyde and primary amines or monosubstituted hydrazines," The Journal of Organic Chemistry, vol. 55, no. 10, pp. 3205-3209, 1990.

[9] C. R. Ganellin and R. G. W. Spickett, "Compounds affecting the central nervous system. I. 4-piperidones and related compounds," Journal of Medicinal Chemistry, vol. 8, no. 5, pp. 619625, 1965.

[10] G. Aridoss, S. Amirthaganesan, N. Ashok Kumar et al., "A facile synthesis, antibacterial, and antitubercular studies of some piperidin-4-one and tetrahydropyridine derivatives," Bioorganic and Medicinal Chemistry Letters, vol. 18, no. 24, pp. 6542-6548, 2008.

[11] V. M. Margaretha, H. Armin, J. L. Ivonne et al., "Novel selective PDE4 inhibitors. 1. Synthesis, structure-activity relationships, and molecular modeling of 4-(3,4-dimethoxyphenyl)2H-phthalazin-1-ones and analogues," Journal of Medicinal Chemistry, vol. 44, no. 16, pp. 2511-2522, 2001.

[12] S. A. Abubshait, "An efficient synthesis and reactions of novel indolylpyridazinone derivatives with expected biological activity," Molecules, vol. 12, no. 1, pp. 25-42, 2007.

[13] P. Coudert, C. Rubat, P. Tronche, P. Bastide, and J. Bastide, "Synthesis and antisecretory and antiulcer activity of novel $\mathrm{N}$ ureidoalkyl pyridazinones," Pharmaceutica Acta Helvetiae, vol. 64, no. 5-6, pp. 159-162, 1989.

[14] A. S. A. Youssef, M. I. Marzouk, H. M. F. Madkour, A. M. A. El-Soll, and M. A. El-Hashash, "Synthesis of some heterocyclic systems of anticipated biological activities via 6-aryl-4-pyrazol1-yl-pyridazin-3-one," Canadian Journal of Chemistry, vol. 83, no. 3, pp. 251-259, 2005.

[15] S.-C. Cherng, W.-H. Huang, C.-Y. Shiau, A.-R. Lee, and T.C. Chou, "Mechanisms of antiplatelet activity of PC-09, a newly synthesized pyridazinone derivative," European Journal of Pharmacology, vol. 532, no. 1-2, pp. 32-37, 2006.

[16] D. S. Doğruer, T. Önkol, S. Özkan, S. Özgen, and M. F. Şahin, "Synthesis and antimicrobial activity of some $3(2 \mathrm{H})$ pyridazinone and 1(2H)-phthalazinone derivatives," Turkish Journal of Chemistry, vol. 32, no. 4, pp. 469-479, 2008.

[17] H. A. Taleb, "Design and synthesis of novel tetrahydro-2HPyrano[3,2-c]Pyridazin-3(6H)- one derivatives as potential anticancer agents," European Journal of Medicinal Chemistry, vol. 45 , no. 12 , pp. 5724-5731, 2010.

[18] I. G. Rathish, J. Kalim, A. Shamim et al., "Synthesis and evaluation of anticancer activity of some novel 6-aryl-2-(psulfamylphenyl)-pyridazin-3(2H)-ones," European Journal of Medicinal Chemistry, vol. 49, pp. 304-309, 2012.

[19] C. R. Noller and V. Baliah, "The preparation of some piperidine derivatives by the Mannich reaction," Journal of the American Chemical Society, vol. 70, no. 11, pp. 3853-3855, 1948.

[20] V. Baliah and A. Ekambaram, "Condensation of levulinic acid and its ethyl ester with aldehydes and ammonia," Science and Culture, vol. 20, p. 193, 1954.

[21] V. Baliah, A. Ekambaram, and T. S. Govindarajan, "Condensation of acetone with aldehydes and ammonia," Current Science, vol. 23, p. 264, 1954.

[22] V. Baliah and R. Jeyaraman, "Rates of esterification of some azabicyclo[3. 3. 1]nonan-9-ols," Indian Journal of Chemistry B: Organic and Medicinal Chemistry, vol. 15, pp. 832-834, 1977.

[23] S. Thennarasu and P. T. Perumal, "An efficient preparation of 1,2diamino-1-phenylheptane," Molecules, vol. 7, no. 6, pp. 487-493, 2002.
[24] W. Wan, J. Hou, H. Jiang et al., "Concise synthesis of $\omega$ fluoroalkylated ketoesters. A building block for the synthesis of six-, seven-, and eight-membered fluoroalkyl substituted 1,2diaza-3-one heterocycles," Tetrahedron, vol. 65, no. 21, pp. 42124219, 2009.

[25] T. Ravindran, R. Jeyaraman, R. W. Murray, and M. Singh, "Chemistry of N-nitroso compounds. 1. Synthesis and stereodynamics of N-nitrosopiperidines and N-nitrosopiperidin-4ones," The Journal of Organic Chemistry, vol. 56, no. 16, pp. 48334840, 1991.

[26] M. Srinivasan, S. Perumal, and S. Selvaraj, "Synthesis, stereochemistry, and antimicrobial activity of 2,6-diaryl-3(arylthio)piperidin-4-ones," Chemical and Pharmaceutical Bulletin, vol. 54, no. 6, pp. 795-801, 2006.

[27] A. Manimekalai, B. S. Sivakumar, and T. Maruthavanan, "NMR spectral studies of some N-aroyl hydrazones," Indian Journal of Chemistry B: Organic and Medicinal Chemistry, vol. 43, no. 8, pp. 1753-1757, 2004.

[28] V. P. A. Raja and S. Perumal, "A tandem multi-component synthesis of 5,7-diaryl-5,6,7,8-tetrahydro-1H-pyrido[3,4b] $[1,4]$ thiazin-2(3H)-ones," Tetrahedron, vol. 62, no. 20, pp. 4892-4899, 2006.

[29] The crystal data of compound $\mathbf{3 b}$ had already been submitted to The Cambridge Crystallographic Data Centre (CCDC no. 902856).

[30] T. J. Mosmann, "Rapid colorimetric assay for cellular growth and survival: application to proliferation and cytotoxicity assays," Journal of Immunological Methods, vol. 65, no. 1-2, pp. 55-63, 1983.

[31] F. Denizot and R. Lang, "Rapid colorimetric assay for cell growth and survival-modifications to the tetrazolium dye procedure giving improved sensitivity and reliability," Journal of Immunological Methods, vol. 89, no. 2, pp. 271-277, 1986. 

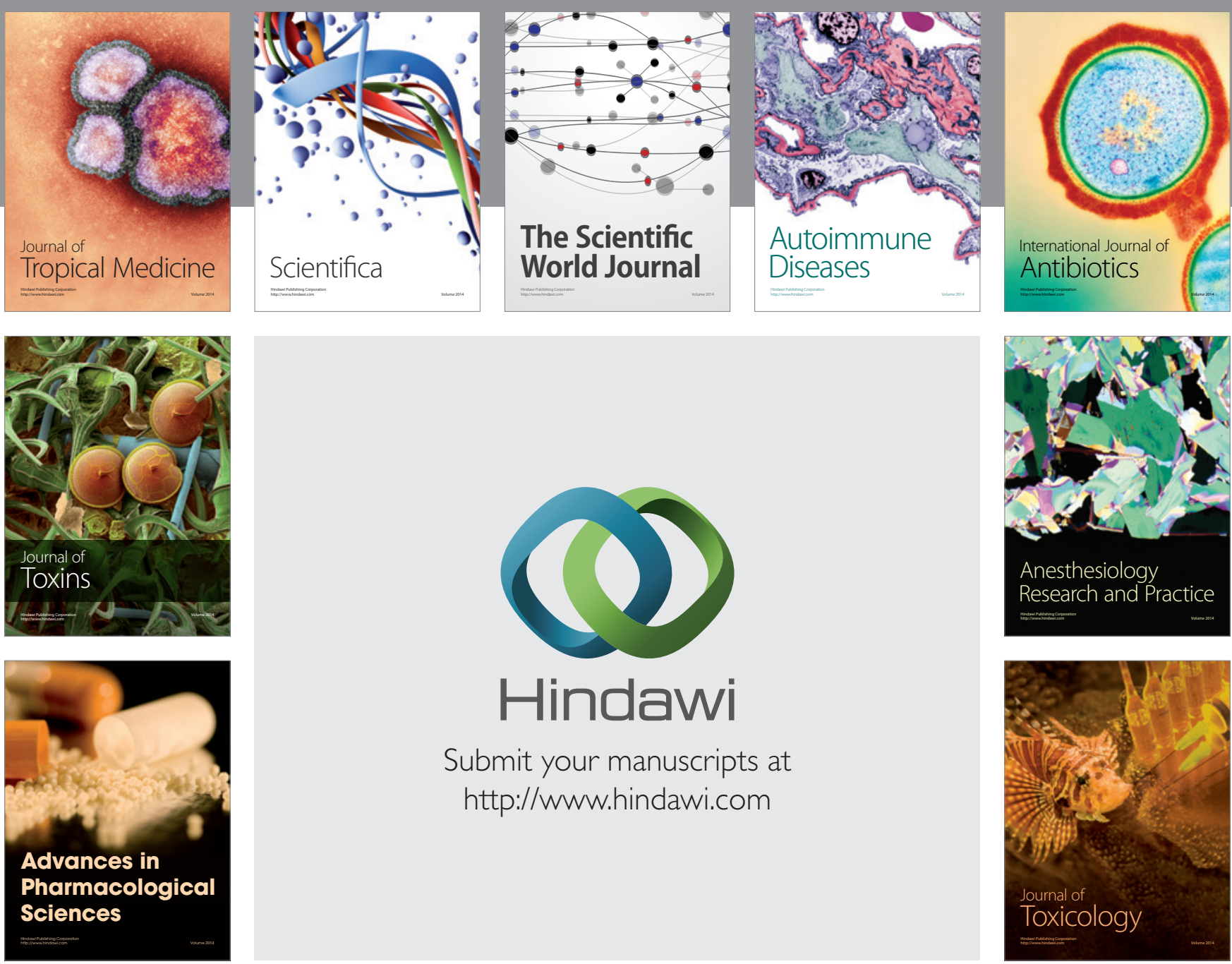

\section{Hindawi}

Submit your manuscripts at

http://www.hindawi.com
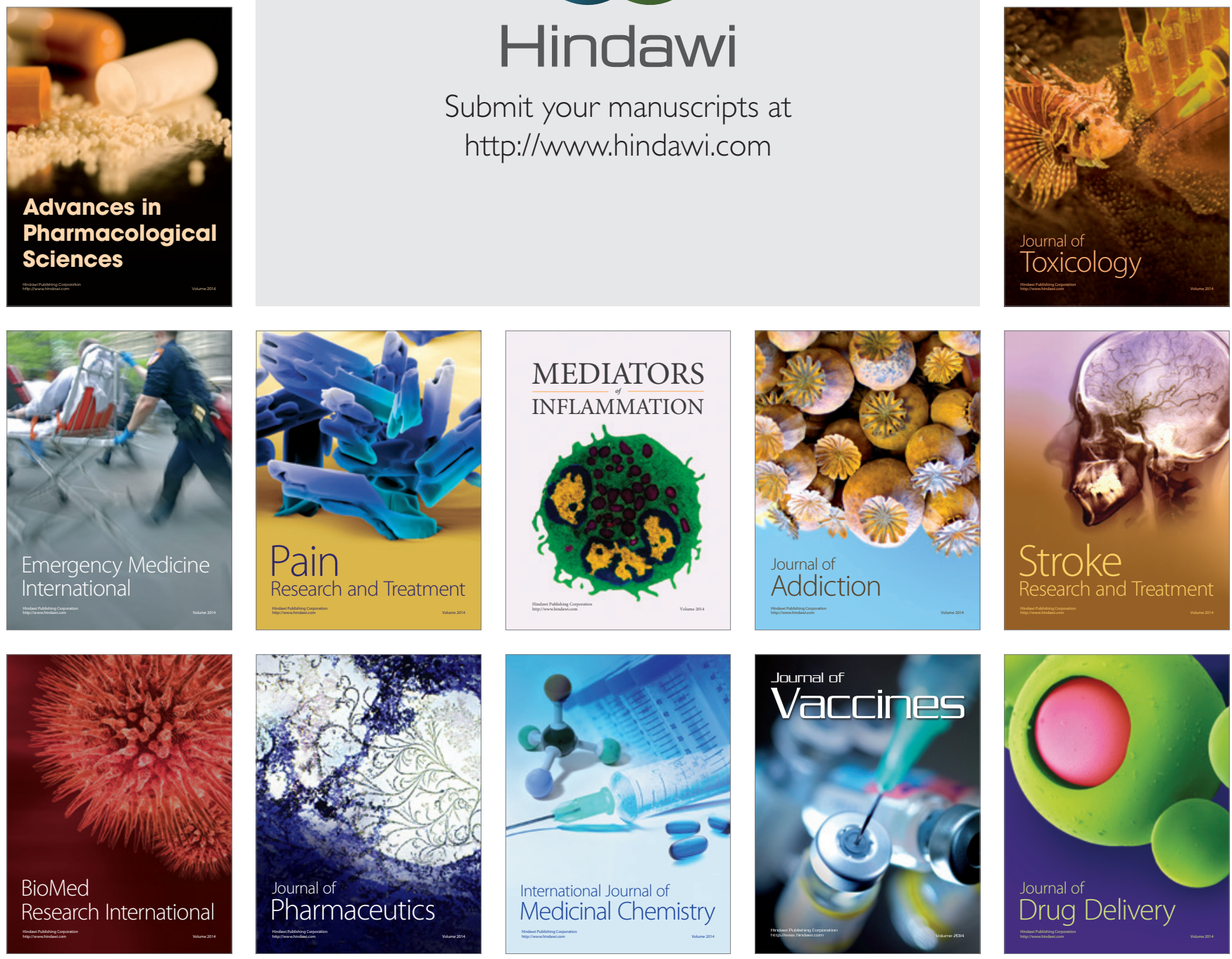